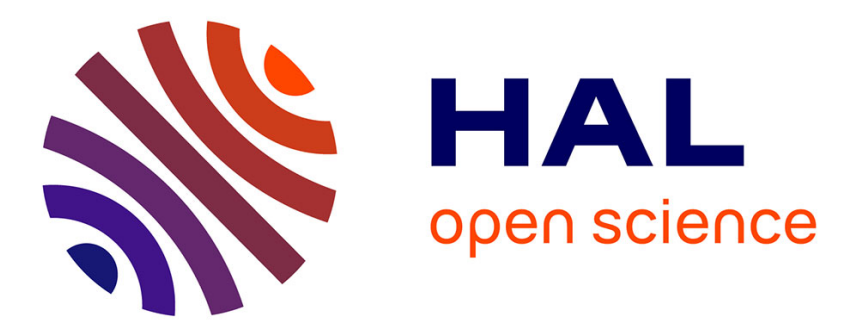

\title{
Photo-Robotic Extrinsic Parameters Calibration of 6-DOF Robot for High Positioning Accuracy
}

Houari Bettahar, Olivier Lehmann, Cédric Clevy, Nadège Courjal, Philippe Lutz

\section{To cite this version:}

Houari Bettahar, Olivier Lehmann, Cédric Clevy, Nadège Courjal, Philippe Lutz. Photo-Robotic Extrinsic Parameters Calibration of 6-DOF Robot for High Positioning Accuracy. IEEE/ASME Transactions on Mechatronics, 2020. hal-02991173

\section{HAL Id: hal-02991173 \\ https://hal.science/hal-02991173}

Submitted on 5 Nov 2020

HAL is a multi-disciplinary open access archive for the deposit and dissemination of scientific research documents, whether they are published or not. The documents may come from teaching and research institutions in France or abroad, or from public or private research centers.
L'archive ouverte pluridisciplinaire HAL, est destinée au dépôt et à la diffusion de documents scientifiques de niveau recherche, publiés ou non, émanant des établissements d'enseignement et de recherche français ou étrangers, des laboratoires publics ou privés. 


\title{
Photo-Robotic Extrinsic Parameters Calibration of 6-DOF Robot for High Positioning Accuracy
}

\author{
Houari Bettahar, Olivier Lehmann, Cédric Clévy, Nadège Courjal and Philippe Lutz
}

\begin{abstract}
Hybrid integration of individual photonic elements appears particularly promising. It indeed provides high performances, proposes new optical functionalities and products and exploits new propagation modes of light beams. This approach requires an accurate multi Degree-Of-Freedom (DOF) positioning of the individual photonic elements. Hence, the inaccurate multiDOF measurements and robots control are the main locks to overcome, notably at the micro-scale. For this sake, an original photo-robotic approach has been proposed. It relies on multi-DOF robot's motion associated with the use of 1-D Fabry-Perot interferometry measurement to achieve multi-DOF pose measurement. This original photo-robotic approach notably integrates the issue of extrinsic parameters calibration through a proposed photo-robotic calibration model. Experimental investigations demonstrate that a translational positioning accuracy of $390 \mathrm{~nm}$ was obtained. This photo-robotic approach has especially been applied to achieve the 6-DOF positioning of an optical lamella relative to an optical fiber with high accuracy that also conducts to maximum optical reflectivity.
\end{abstract}

Index Terms-Nano-positioning, Accuracy, Photo-robotic, robot calibration, Photonics.

\section{INTRODUCTION}

Achieving fast, automated 3D complex tasks in micromanipulation and micro-assembly with very high accuracy is currently strongly desired in many application fields, such as advanced integrated optics manufacturing. This would indeed make it possible to achieve commercial success with reliable and cost-effective assembly, and packaging technologies. Many emerging industrial on-demand applications made the field of integrated optics particularly challenging, because of their increasing functions and complexity. These applications require the integration of various building components fabricated from different technologies in order to achieve a wide rang of microsystems. The possibility to achieve hybrid nano-photonic components by assembly feeds many original applications. It also brings very relevant solutions to overcome the monolithic integration difficulties as well as to achieve 3D multi functions optical devices manufactured with different technologies [1], [2], [3].

Micro-assembly also aims at optimizing the optical performances. Moreover, it enables the development of complex integrated optical devices, from the assembly of different optical components that then form an integrated optical system.

\footnotetext{
H.Bettahar, O.Lehmann, C.Clévy, N.Courjal, P.Lutz are with FEMTO-ST Institute, Univ. Bourgogne Franche-Comté, UFC/CNRS/ENSMM, 24 rue Savary, 25000 Besançon, France, (e-mails: houari.bettaharefemto-st.fr, olivier.lehmanndemto-st.fr, cclevy@femto-st.fr, philippe.lutz@femto-st.fr, nadege.courjaldfemto-st.fr)
}

Positioning and assembly for integrated optics industry are generally done manually based on passive and active positioning [4] [5] [6]. In passive positioning, operator may use geometrical devices such as V-grooves [4]. This kind of approach enables to reach a positioning accuracy of about $1 \mu \mathrm{m}$ [7], it is accurate enough for many applications as demonstrated for passive alignment of two photonic chips on a silicon optical bench [8]. There are numerous very accurate machines like flip-chip [9] [10] that are used in integrated optics industry. However, these technologies are limited to a specific type of products. For complex 3D tasks, positioning based on visual control is usually achieved by teleoperated way in air [11]. These approaches are more generic, a positioning accuracy of about $10 \mu \mathrm{m}$ has been achieved [12].

However, all these cited strategies rely on geometrical features to relatively position two components relative to one another, the optical performances are not guaranteed and the positioning accuracy is not sufficient. For this sake, the active approach is the mainly used one for integrated optics industry, since it maximizes the optical irradiance during the positioning task. Micro and nano-positioning systems are used to adjust the position of the optical component manually or in a teleoperated way[13] in order to obtain for instance, the maximum optical irradiance. However, it requires dexterity and skills from the operator along with a lot of precision and attention as it is a tiring task for the operator. The operator applies a non-referenced motion particularly for multi-DOF motion which leads to a local maximum problem on the obtained optical irradiance. The repeatability and accuracy of the positioning may reach some hundreds of $\mathrm{nm}$ but at a high cost due to the training of the human operator and the long time needed to achieve the task. Consequently, the performances and the quality of the assembled products are strongly operator-dependent.

Micro-robotic approaches enable to position a frame of a robot, typically an end-effector to the desired location through referenced robotic motions with respect to already attributed reference frames. The extrinsic geometric parameters are the parameters relating the robot end-effector to tool frame, world frame and measurement system frame. The robot end-effector position control is achieved by using the Direct or the Inverse Geometric Model (DGM or IGM) and the transformation matrices between frames. To successfully perform tasks at micro-scale, micro-systems or micro-robots with high positioning accuracy are required. However, this requirement is difficult to obtain at the micro-scale because of many extrinsic geometric parameters acting on micro-robots [14], [15]. A solution would be to use additional sensors to provide an 
outer-loop control (the often used inner-loop feedback one is based on internal sensors of the stages) [16]. However, a key limitation is the complexity of integrating sensors able to measure a relative position with required range, resolution, bandwidth and number of DOF (Degrees-Of-Freedom) [17].

For this sake, works intend to overcome the active positioning drawbacks by exploiting the high accurate 1-D interferometric measurements that are particularly and naturally produced during classical active positioning, called Fabry-Perot interferences. The aim is then to associate these measurements with a multi-DOF referenced robotic motion. The core objective of this proposed approach is to achieve high accurate multi-DOF positioning in order to get maximum optical performances. In [18], a very preliminary study was conducted considering two optical building components (fiber ferrule, optical component). The study demonstrated that identifying only two parameters (misalignment angles) can largely improve optical performances. This work also stated the potential of a new approach named Photo-robotic because it merges and then correlates the optical, mechanical and robotic behaviors. This work also showed the need to establish a theoretical model and an associated measurement and identification strategy in a formal and generic way to consider more and different types of parameters. For these reasons, this paper aims at generalizing the photo-robotic approach to calibrate all extrinsic geometric parameters of the robot. A new photo-robotic calibration model and an associated measurement strategy are especially proposed. It combines a 1-D Fabry-Perot interferometric measurement with the motions of a multi-DOF robot. Once the robot extrinsic parameters identified, the optical lamella, which is attached to the robot, can be positioned in 6-DOF with very high accuracy. The paper is organized as follows. Section II explains the Fabry-Perot interferometry principle. Section III models geometrically the 6-DOF robot used for positioning. Section IV presents the proposed photo-robotic calibration model. Section $\mathrm{V}$ presents the experimental procedure for robot extrinsic parameters calibration. Section VI presents the experimental setup. Section VII investigates the behavior of the calibrated extrinsic parameters of the robot. Section VIII concludes the paper.

\section{FABRY-PEROT INTERFEROMETRY}

Interferometer sensors are very attracting to use due to their high resolution, accuracy, wide measuring range and bandwidth. These interferometer sensors can be operating based on different interferometry principles such as Michelson and Fabry-Perot interferometry. Active alignment of the optical lamella with respect to the optical fiber produces the Fabry-Perot interferometry phenomenon. The Fabry-Perot interferometry results from the phenomenon of multiple beam interferences that arises when light shines through a cavity bounded by two reflective surfaces as shown in Figure 1 In our case, the involved surfaces are the fiber extremity $\left(R_{1}\right)$ in one hand and the lamella surface $\left(R_{2}\right)$ in the other hand. Each time the light encounters one of the surfaces, a portion of it is transmitted out, and the remaining part is reflected

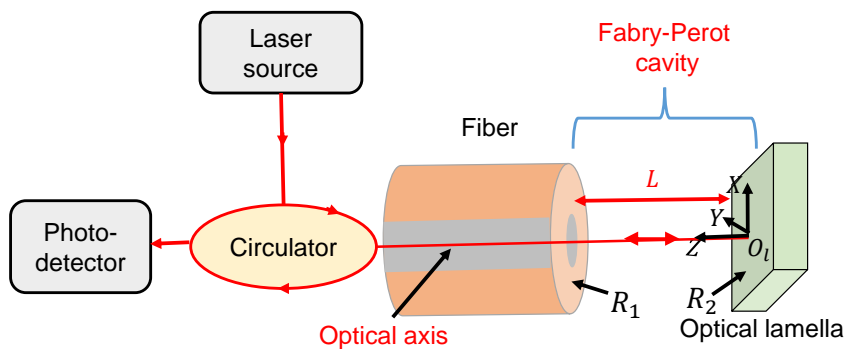

Figure 1. Schematic diagram representing the interferences in a Fabry-Perot optical cavity.

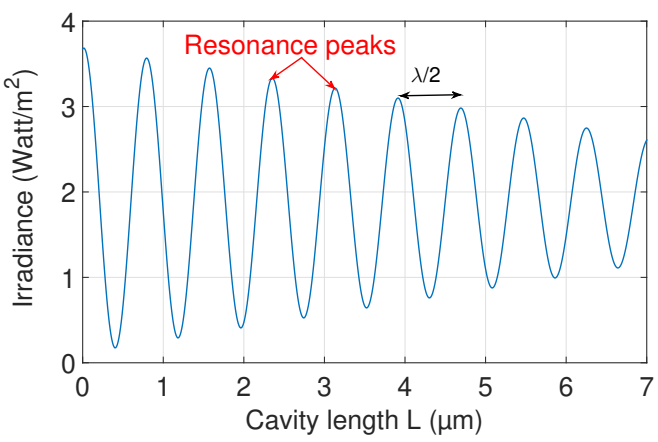

Figure 2. Reflected irradiance versus Fabry-Perot (FP) cavity length $L$, at wavelength $\lambda=1560 \mathrm{~nm}$.

back. The net effect is to split a single beam into multiple beams which interfere with each other. The system behaves like the Fabry-Perot interferometer, where interferences result from the recombination of multiple reflections between the reflective surfaces $R_{1}$ and $R_{2}$ respectively.

Based on Fabry-Perot interferometry principle, when the reflective surface $\left(R_{2}\right)$ moves along the optical axis (Z-axis), the Fabry-Perot cavity length $(L)$ changes and modifies the reflected light signal accordingly. Therefore, there is a correlation between the FP cavity length $L$ and the interference figure. A slight variation of the FP cavity length can be identified on the interference figure.

The periodicity of the irradiance signal directly depends on the wavelength of the laser signal $(\lambda=1560 \mathrm{~nm})$. If $p$ is a natural number, the distance between two resonances can be written as in equation 11 (see Figure 2), i.e. the distance variation between two peaks $(\delta L)$ is a multiple of half wavelength $\lambda$ of the laser irradiance, such as :

$$
\delta L=\frac{\lambda}{2} \cdot p \quad \text { with } \quad p \in \mathbf{N}
$$

Active positioning and assembly of optical components gives the opportunity to measure a high accurate 1-D relative position along the optical axis with very high resolution. This 1-D Fabry-Perot measure is chosen to be used as a feedback information for positioning of the optical lamella with respect to the fiber. From the other hand, active positioning of optical components generally uses different positioning systems in order to multi-DOF motion, each positioning system realizes motion with respect to it own actuation frame. This leads to a non-referenced multi-DOF motion. The DGM is used to 
control the different positioning systems as one robot in order to calculate the robot end-effector coordinates with respect to a fixed reference frame as a function of the joints coordinates. The DGM modeling of our 6-DOF robot is the objective of the next section.

\section{6-DOF ROBOT GEOMETRIC MODELING}

Geometric model deals with the relationship between the joint and operational coordinates (coordinates with respect to the world reference frame), by considering robot geometric parameters. Geometric model enables to establish of the robot DGM (Direct Geometric Model) and IGM (Inverse Geometric Model), which are successively used to control the robot. It usually consists in determining mathematical description that describes the required coordinate transformations based on different approaches for the geometric representation. One of the most known method has been accomplished by Denavit and Hartenberg [19]. The method based on homogeneous transformation matrices. The procedure consists of establishing coordinate systems on each joint axis. Each coordinate system is then related to the next through a specific set of parameters in the homogeneous transformation matrices. Afterwards, many researchers mentioned the model singularity problem raised in D-H representation[20]. A new notation has been derived from the popular $\mathrm{D}-\mathrm{H}$ method by Khalil and Kleinfinger [21] which can be used to describe the openloop robots and the closed-loop robots with a minimum of parameters and without ambiguities or difficulties. Khalil and Kleinfinger representation is one of the most used conventions for the geometric models. However, in the case of nearly parallel neighboring axes, the common normal is poorly defined and the calibration is ill-conditioned. For this case, Hayati introduced an extra rotational parameter $\beta_{i}$ about the $Y_{(} i-1$ axis [22].

The modified version by Khalil and Kleinfinger with additional Hayati parameter $\beta_{i}$ is used to establish the transformation matrix between two successive links based on two main assumptions: the links are perfectly rigid and the joints are ideal in a sense that there is neither backlash nor elasticity. A serial robot is composed of a sequence of $n$ links and $\mathrm{n}$ joints where link 0 is the base of the robot and link $n$ is the terminal link. Joint $i$ connects the link $i$ to the link $i-1$ and its variable is denoted by $\theta_{i}$. A frame $R_{i}$ is attached to each link $i$ with [23]:

- The $Z_{i}$ axis is located along the axis of joint $i$;

- The $X_{i}$ axis is located along the common normal between the $Z_{i}$ and $Z_{i+1}$ axes. If $Z_{i}$ and $Z_{i+1}$ axes are parallel or collinear, the choice of $X_{i}$ is not unique: considerations of symmetry or simplicity then allow a rational choice;

- The $Y_{i}$ axis is located using the right-hand rule to form $\left(X_{i}, Y_{i}, Z_{i}\right)$ coordinate system.

The transformation matrix from frame $R_{i-1}$ to frame $R_{i}$ is expressed using the following four geometric parameters (Figure 3):

- Joint parallelism: $\beta_{i}$ is the angle around the axis $Y_{i-1}$ between the axes $Z_{i-1}$ and $Z_{i-1}^{\prime}$; $\mathrm{h}$

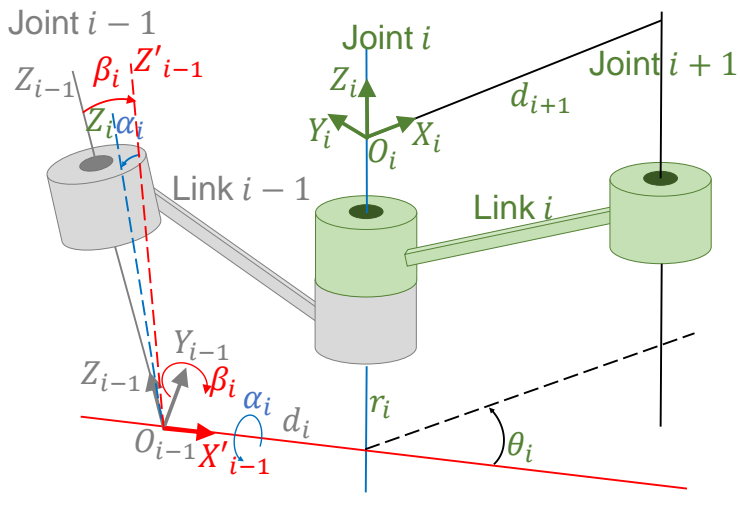

Figure 3. The additional Hayati parameter $\beta_{i}$ about $Y_{i-1}$ is employed for nearly parallel axes.

- Link twist: $\alpha_{i}$ is the angle around the axis $X_{i-1}^{\prime}$ and between the axes $Z_{i-1}^{\prime}$ and $Z_{i}$;

- Link length: $d_{i}$ is the distance along the axis $X_{i-1}^{\prime}$ between the axes $Z_{i-1}^{\prime}$ and $Z_{i}$;

- Joint angle: $\theta_{i}$ is the angle around the axis $Z_{i}$ between the axes $X_{i-1}^{\prime}$ and $X_{i}$;

- Link offset: $r_{i}$ is the distance along the axis $Z_{i}$ between the axes $X_{i-1}^{\prime}$ and $X_{i}$.

The variable of the joint $i$ denoted by $q_{i}$ is $\theta_{i}$ if $i$ is rotational and $r_{i}$ if $i$ is prismatic. Hence

$$
q_{i}=\theta_{i}\left(1-\rho_{i}\right)+r_{i} \rho_{i}
$$

where

$$
\rho_{i}= \begin{cases}0, & \text { for rotational joint } \\ 1, & \text { for prismatic joint }\end{cases}
$$

Two rotations are required to relate $Z_{i-1}$ to $Z_{i}$ : a rotation $\beta_{i}$ about $Y_{i-1}$ maps $Z_{i-1}$ to $Z_{i-1}^{\prime}$ and $X_{i-1}$ to $X_{i-1}^{\prime}$. Hence, $X_{i-1}^{\prime}$ axis is located along the common normal between the $Z_{i-1}^{\prime}$ and $Z_{i}$ axes. A second rotation $\alpha_{i}$ about $X_{i-1}^{\prime}$ maps $Z_{i-1}^{\prime}$ to $Z_{i}$. The angle $\theta_{i}$ is now from $X_{i-1}^{\prime}$ to $X_{i}$ about $Z_{i}$.

The transformation matrix defining the frame $R_{i}$ in the frame $R_{i-1}$ is given by

$$
\begin{aligned}
& { }^{i-1} T_{i}=\operatorname{Rot}_{Y_{i-1}}\left(\beta_{i}\right) \cdot \operatorname{Rot}_{X_{i-1}^{\prime}}\left(\alpha_{i}\right) \cdot \operatorname{Trans}_{X_{i-1}^{\prime}}\left(d_{i}\right) \\
& \operatorname{Rot}_{Z_{i}}\left(\theta_{i}\right) \cdot \operatorname{Trans}_{Z_{i}}\left(r_{i}\right)
\end{aligned}
$$

DGM can be obtained by simply concatenating the transformations between the fixed frames of the adjacent links [23]:

$$
{ }^{0} T_{n}(q)={ }^{0} T_{1}\left(q_{1}\right) \cdot{ }^{1} T_{2}\left(q_{2}\right) \ldots{ }^{n-2} T_{n-1}\left(q_{n-1}\right) \cdot{ }^{n-1} T_{n}\left(q_{n}\right)
$$

The inverse problem is to calculate the joint coordinates corresponding to a given operational coordinates of the endeffector. When exists, the form that gives all the possible solutions (there is rarely a single solution) is The Inverse Geometric Model (IGM).

Our objective is to calibrate the extrinsic geometric parameters of the robot. Hence, the intrinsic parameters of the robot are assumed nominals. For this sake, the same origin for all the reference frames is chosen, which corresponds to the real 


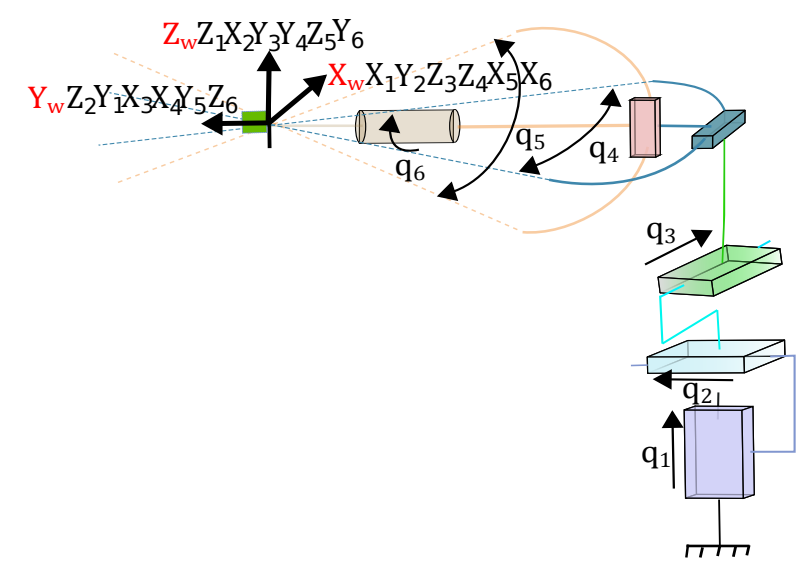

Figure 4. Robot kinematic model.

Table I

NOMINAL PARAMETERS OF DGM OF THE ROBOT BASED ON HAYATI MODIFIED CONVENTION OF KHALIL AND KLEINFINGER.

\begin{tabular}{ccccccc}
\hline Link & $\rho_{i}$ & $\beta_{i}$ & $\alpha_{i}$ & $d_{i}$ & $\theta_{i}$ & $r_{i}$ \\
\hline 1 & 1 & 0 & 0 & 0 & 0 & $q_{1}$ \\
2 & 1 & 0 & -90 & 0 & -90 & $q_{2}$ \\
3 & 1 & 0 & -90 & 0 & -90 & $q_{3}$ \\
4 & 0 & 0 & 0 & 0 & $q_{4}$ & 0 \\
5 & 0 & 0 & -90 & 0 & $q_{5}-90$ & 0 \\
6 & 0 & 0 & -90 & 0 & $q_{6}$ & 0 \\
\hline
\end{tabular}

rotational center of the robot (intersection of joint 4 and 5) when $q_{1}=q_{2}=q_{3}=q_{4}=q_{5}=q_{6}=0$. The world (base) frame $R_{w}$ is assigned to be with the same orientations as the first frame when $q_{1}=0$. The nominal geometric parameters of the robot are then determined. The assignment of frames is shown in Figure 4, which represents the robot geometric model and the geometrical parameters for each link are given in Table 【.

\section{ROBOT EXTRINSIC PARAMETERS CALIBRATION BASED ON 1-D FABRY-PEROT RELATIVE DISTANCE MEASURE}

The objective of this section is to achieve the robot extrinsic geometric calibration based on relative distance measure. The distance measure is realized based on very high accurate Fabry-Perot interferometry principle measurement as presented in section II. In order to go through the calibration process, the main reference frames are defined as schematically in Figure 5. $R_{f}$ is assigned to the fiber frame, its origin, labeled $O_{f}$, is defined as the intersection point between the optical axis and the outer surface of the fiber. $R_{e}$ corresponds to the robot end-effector frame, its origin, labeled $O_{e}$, corresponds to the real rotational center of the robot (intersection of joint 4 and 5). The $\overrightarrow{O_{e} X_{e}}, \overrightarrow{O_{e} Y_{e}}$ and $\overrightarrow{O_{e} Z_{e}}$ are the expected motion directions of the robot. $R_{t}$ corresponds to the tool frame, its origin, labeled $O_{t}$, is defined as the intersection between the $\overline{O_{e} Z_{e}}$ and the optical lamella plane $\left(Y_{t} O_{t} X_{t}\right) . R_{w}$ is assigned to the world frame, its origin, labeled $O_{w}$, corresponds to the real rotational center of the robot (intersection of joint 4 and 5) when all axes are at zero (initial configuration), with the same directions as the first stage axes $R_{1}$.

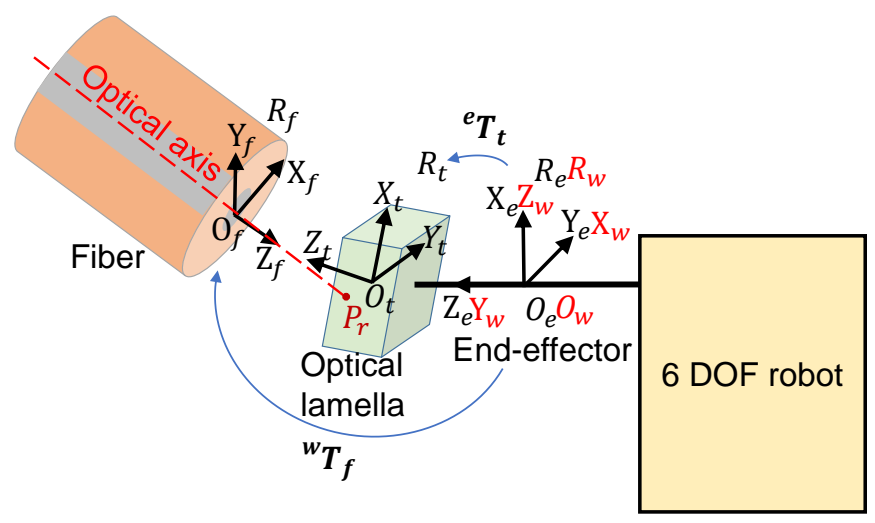

Figure 5. The photo-robotic positioning scheme with frames assignment.

For simplification, the rotation of the fiber around $Y_{w}$ is assumed to be zero, the rotation of the lamella around $Z_{e}$ axis and the translations of the lamella along $X_{e}$ and $Y_{e}$ axis all are supposed to be zero. The vector $\overrightarrow{O_{e} Z_{e}}$ is supposed to be collinear with the vector $\overrightarrow{O_{w} Y_{w}}$. Thus, the homogeneous transformation matrix from the world frame to the fiber frame is given in equation (6), and the transformation matrix from the end-effector frame to the tool frame is given in equation (7).

$$
\begin{aligned}
& { }^{w} T_{f}=\operatorname{Trans}_{X_{w}}\left(x_{w f}\right) \cdot \operatorname{Trans}_{Y_{w}}\left(y_{w f}\right) . \text { Trans }_{Z_{w}}\left(z_{w f}\right) \\
& . \operatorname{Rot}_{X_{w}}\left(\beta_{w f}\right) * \operatorname{Rot}_{Z_{w}}\left(\theta_{w f}\right)= \\
& {\left[\begin{array}{cccc}
\cos \left(\theta_{w f}\right) & -\sin \left(\theta_{w f}\right) & 0 & x_{w f} \\
\cos \left(\beta_{w f}\right) \sin \left(\theta_{w f}\right) & \cos \left(\theta_{w f}\right) \cos \left(\beta_{w f}\right) & -\sin \left(\beta_{w f}\right) & y_{w f} \\
\sin \left(\beta_{w f}\right) \sin \left(\theta_{w f}\right) & \cos \left(\theta_{w f}\right) \sin \left(\beta_{w f}\right) & \cos \left(\beta_{w f}\right) & z_{w f} \\
0 & 0 & 0 & 1
\end{array}\right]}
\end{aligned}
$$

where $x_{w f}, y_{w f}$ and $z_{w f}$ are the translational coordinates of the origin of the fiber frame $O_{f}$ with respect to the world frame, along $X_{w}, Y_{w}$ and $Z_{w}$ axis respectively. $\beta_{w f}$ is the angle between $Y_{f}$ and $Z_{w}$ around $X_{w}$ axis and $\theta_{w f}$ is the angle between $X_{f}$ and $X_{w}$ around $Z_{w}$ axis.

$$
\begin{aligned}
& { }^{e} T_{t}=\operatorname{Trans}_{Z_{e}}\left(Z_{e t}\right) \cdot \operatorname{Rot}_{X_{e}}\left(\beta_{e t}\right) \cdot \operatorname{Rot}_{Y_{e}}\left(\theta_{e t}\right)= \\
& {\left[\begin{array}{cccc}
\cos \left(\theta_{e t}\right) & 0 & \sin \left(\theta_{e t}\right) & 0 \\
\sin \left(\theta_{e t}\right) \sin \left(\beta_{e t}\right) & \cos \left(\beta_{e t}\right) & -\cos \left(\theta_{e t}\right) \sin \left(\beta_{e t}\right) & 0 \\
-\cos \left(\beta_{e t}\right) \sin \left(\theta_{e t}\right) & \sin \left(\beta_{e t}\right) & \cos \left(\theta_{e t}\right) \cos \left(\beta_{e t}\right) & Z_{e t} \\
0 & 0 & 0 & 1
\end{array}\right]}
\end{aligned}
$$

where $Z_{e t}$ is the translational coordinate of the origin of the optical lamella $O_{t}$ with respect to the end-effector frame, along $Z_{e}$ axis. $\beta_{e t}$ and $\theta_{e t}$ are the rotational coordinates of $X_{t}$ and $Y_{t}$ axes of the optical lamella with respect to the end-effector frame, around the $X_{e}$ and $Y_{e}$ respectively.

$P_{r}$ is the reflection point projection of the laser spot on the optical lamella plane $\left(Y_{t} O_{t} X_{t}\right) .{ }^{w} P_{r}\left({ }^{w} P_{r x},{ }^{w} P_{r y},{ }^{w} P_{r z}\right)$ is the reflection point projection in the world frame $R_{w}$. ${ }^{t} P_{r}\left({ }^{t} P_{r x},{ }^{t} P_{r y}, 0\right)$ is the reflection point in the tool frame $R_{t}$.

All the required transformations are defined, and they are used for the proposed extrinsic parameters calibration.

The identification step of the proposed extrinsic geometric parameters calibration consists of three steps: 
A. Identification of the optical lamella plane orientation and the optical axis orientation with respect to the world frame $\left(\beta_{e t}, \theta_{e t}, \beta_{w f}, \theta_{w f}\right)$

The parametric form of the equation of optical axis line is defined by a point on the optical axis line $O_{f}$ and its direction vector $\overrightarrow{Z_{f}}$. The point $O_{f}\left(x_{w f}, y_{w f}, z_{w f}\right)$ is a point with respect to the world frame $R_{w}$ through which the optical axis line passes. The unit vector $\overrightarrow{Z_{f}}\left(a_{w f}, b_{w f}, c_{w f}\right)$ is the direction vector of the optical axis line with respect to the world frame $R_{w}$.

The 3-dimensional parametric equations of the line carrying the vector $\overrightarrow{O_{f} Z_{f}}$ (optical axis) can be written as in equation. (8):

$$
\left\{\begin{array}{l}
x=a_{w f} L+x_{w f} \\
y=b_{w f} L+y_{w f} \\
z=c_{w f} L+z_{w f}
\end{array}\right.
$$

where $\sqrt{a_{w f}^{2}+b_{w f}^{2}+c_{w f}^{2}}=1$.

The intersection point between the lamella plane $\left(Y_{t} O_{t} X_{t}\right)$ and the line carries the vector $\overrightarrow{O_{e} Z_{e}}$ is $O_{t}\left(x_{w t}, y_{w t}, z_{w t}\right)$. The optical lamella plane is defined by the point $O_{t}\left(x_{w t}, y_{w t}, z_{w t}\right)$ in the plane with respect to the world frame and the normal unit vector to the plane $\vec{Z}_{t}\left(a_{w t}, b_{w t}, c_{w t}\right)$. If $M(x, y, z)$ is any other point which belongs to the lamella plane, then the equation of the lamella plane can be written as follows:

$$
\overrightarrow{O_{t} M} \cdot \overrightarrow{O_{t} Z_{t}}=0
$$

This yields:

$$
a_{w t}\left(x-x_{w t}\right)+b_{w t}\left(y-y_{w t}\right)+c_{w t}\left(z-z_{w t}\right)=0
$$

By considering $d_{w t}=-a_{w t} x_{w t}-b_{w t} y_{w t}-c_{w t} z_{w t}$, then:

$$
a_{w t} x+b_{w t} y+c_{w t} z+d_{w t}=0
$$

where: $\sqrt{a_{w t}^{2}+b_{w t}^{2}+c_{w t}^{2}}=1$.

Differentiating the 3 dimensional optical axis line equation (8) and the optical lamella plane equation (11) at the intersection point between the optical axis line and the lamella plane respectively gives :

$$
\begin{gathered}
\left\{\begin{array}{c}
d x=a_{w f} d L \\
d y=b_{w f} d L \\
d z=c_{w f} d L
\end{array}\right. \\
a_{w t} d x+b_{w t} d y+c_{w t} d z+d d_{w t}=0
\end{gathered}
$$

Then by replacing the differentiated optical axis line equation (12) in the differentiated optical lamella plane equation (13) and by replacing $d d_{w t}$, the equation is yielded:

$$
a_{w t} a_{w f} d L+b_{w t} b_{w f} d L+c_{w t} c_{w f} d L=a_{w t} d x+b_{w t} d y+c_{w t} d z
$$

Equation (14) is a non-linear equation relating the relative distance along the optical axis with the 3 relative robot translations. The $d x, d y$ and $d z$ are calculated from the Direct Geometric Model (DGM), $d L$ is calculated using Fabry-Perot measure.

The dedicated measurement strategy for identification of the coordinates of the unit vector $\vec{Z}_{f}\left(a_{w f}, b_{w f}, c_{w f}\right)$ and the coordinates of the unit vector $\vec{Z}_{t}\left(a_{w t}, b_{w t}, c_{w t}\right)$ is discussed in the next section.

In order to calculate the $\left(\beta_{e t}, \theta_{e t}, \beta_{w f}, \theta_{w f}\right)$ angles, the mathematical relationship between the identified $\left(a_{w t}, b_{w t}\right.$, $\left.c_{w t}, a_{w f}, b_{w f}, c_{w f}\right)$ and the $\left(\beta_{e t}, \theta_{e t}, \beta_{w f}, \theta_{w f}\right)$ angles need to be established.

The columns of a rotation matrix are orthogonal unit vectors along $X, Y$ and $Z$ axes respectively. Hence, the third column of a rotation matrix represents the unit vector along $Z$-axis. Accordingly, the transformation matrix ${ }^{w} T_{t}$ of the tool frame in the world frame can be written using the unit vector $\vec{Z}_{t}\left(a_{w t}, b_{w t}, c_{w t}\right)$ with respect to the world frame as follows:

$$
{ }^{w} T_{t}={ }^{w} T_{e} \cdot{ }^{e} T_{t}=\left[\begin{array}{cccc}
r & r & a_{w t} & r \\
r & r & b_{w t} & r \\
r & r & c_{w t} & r \\
0 & 0 & 0 & 1
\end{array}\right]
$$

The label $r$ is assigned to the matrix elements that are not required in the development of our approach.

Similarly the the transformation matrix ${ }^{e} T_{t}$ of the tool frame in the end-effector frame can be written using the unit vector $\vec{Z}_{t}\left(a_{e t}, b_{e t}, c_{e t}\right)$ with respect to the end-effector frame as follows:

$$
{ }^{e} T_{t}=\left[\begin{array}{cccc}
r & r & a_{e t} & 0 \\
r & r & b_{e t} & 0 \\
r & r & c_{e t} & Z_{e t} \\
0 & 0 & 0 & 1
\end{array}\right]
$$

The transformation matrix ${ }^{w} T_{e}$ which represents the Direct Geometric Model of the robot (DGM) is supposed nominal. Thus, when $q_{1}=q_{2}=q_{3}=q_{4}=q_{5}=q_{6}=0$, the yielded ${ }^{w} T_{e}$ is given:

$$
{ }^{w} T_{e}=\left[\begin{array}{cccc}
1 & 0 & 0 & 0 \\
0 & 0 & 1 & 0 \\
0 & -1 & 0 & 0 \\
0 & 0 & 0 & 1
\end{array}\right]
$$

By substituting equation (7) and equation (17) in equation (15), this yields:

$$
\begin{aligned}
& { }^{w} T_{t}={ }^{w} T_{e} \cdot{ }^{e} T_{t}= \\
& {\left[\begin{array}{cccc}
\cos \left(\theta_{e t}\right) & 0 & \sin \left(\theta_{e t}\right) & 0 \\
-\cos \left(\beta_{e t}\right) \sin \left(\theta_{e t}\right) & \sin \left(\beta_{e t}\right) & \cos \left(\theta_{e t}\right) \cos \left(\beta_{e t}\right) & Z_{e t} \\
-\sin \left(\beta_{e t}\right) \sin \left(\theta_{e t}\right) & -\cos \left(\beta_{e t}\right) & \cos \left(\theta_{e t}\right) \sin \left(\beta_{e t}\right) & 0 \\
0 & 0 & 0 & 1
\end{array}\right]=} \\
& {\left[\begin{array}{cccc}
r & r & a_{w t} & r \\
r & r & b_{w t} & r \\
r & r & c_{w t} & r \\
0 & 0 & 0 & 1
\end{array}\right]}
\end{aligned}
$$

By substituting equation (16) and equation (17) in equation (15). This yields:

$$
{ }^{w} T_{t}=\left[\begin{array}{cccc}
r & r & a_{e t} & r \\
r & r & c_{e t} & r \\
r & r & -b_{e t} & r \\
0 & 0 & 0 & 1
\end{array}\right]=\left[\begin{array}{cccc}
r & r & a_{w t} & r \\
r & r & b_{w t} & r \\
r & r & c_{w t} & r \\
0 & 0 & 0 & 1
\end{array}\right]
$$

By matching, we deduce:

$$
a_{e t}=a_{w t} ; \quad b_{e t}=-c_{w t} ; \quad c_{e t}=b_{w t} \quad \text { (will be }
$$

used for the 2nd step).

And by matching, we deduce:

$$
\left\{\begin{array}{l}
a_{w t}=\sin \theta_{e t} \\
b_{w t}=\cos \theta_{e t} \cos \beta_{e t} \\
c_{w t}=\cos \theta_{e t} \sin \beta_{e t}
\end{array}\right.
$$


Then, the optical lamella plane orientation angles with respect to the robot end-effector frame can be easily calculated as follow:

$$
\left\{\begin{array}{l}
\beta_{e t}=\operatorname{atan} 2\left(c_{w t}, b_{w t}\right) \\
\theta_{e t}=\operatorname{atan} 2\left(a_{w t} \cos \beta_{e t}, b_{w t}\right)
\end{array}\right.
$$

where $\operatorname{atan} 2(b, a)$ is arc tangent of the two variables $a$ and $b$. It is similar to calculating the arc tangent of $b / a$, except that the signs of both arguments are used to determine the quadrant of the result, which lies in the range $[\pi, \pi][24]$.

With the same way, using equation $22, \beta_{w f}, \theta_{w f}$ can be deduced by matching.

$$
\begin{aligned}
& { }^{w} T_{f}= \\
& {\left[\begin{array}{cccc}
\cos \left(\theta_{w f}\right) & -\sin \left(\theta_{w f}\right) & 0 & X_{w f} \\
\cos \left(\beta_{w f}\right) \sin \left(\theta_{w f}\right) & \cos \left(\theta_{w f}\right) \cos \left(\beta_{w f}\right) & -\sin \left(\beta_{w f}\right) & Y_{w f} \\
\sin \left(\beta_{w f}\right) \sin \left(\theta_{w f}\right) & \cos \left(\theta_{w f}\right) \sin \left(\beta_{w f}\right) & \cos \left(\beta_{w f}\right) & Z_{w f} \\
0 & 0 & 0 & 1
\end{array}\right]} \\
& =\left[\begin{array}{cccc}
r & a_{w f} & r & r \\
r & b_{w f} & r & r \\
r & c_{w f} & r & r \\
0 & 0 & 0 & 1
\end{array}\right]
\end{aligned}
$$

which gives:

$$
\left\{\begin{array}{l}
a_{w f}=-\sin \theta_{w f} \\
b_{w f}=\cos \theta_{w f} \cos \beta_{w f} \\
c_{w f}=\cos \theta_{w f} \sin \beta_{w f}
\end{array}\right.
$$

then the optical axis orientation angles with respect to the world frame can be calculated as follow:

$$
\left\{\begin{array}{l}
\beta_{w f}=\operatorname{atan} 2\left(c_{w f}, b_{w f}\right) \\
\theta_{w f}=\operatorname{atan} 2\left(-a_{w f} \cos \beta_{w f}, b_{w f}\right)
\end{array}\right.
$$

B. Identification of the Cartesian coordinates of the two origins $O_{t}\left(0,0, Z_{e t}\right)$ and $O_{f}\left(x_{w f}, y_{w f}, z_{w f}\right)$ of the optical lamella and the fiber respectively with respect to the world frame

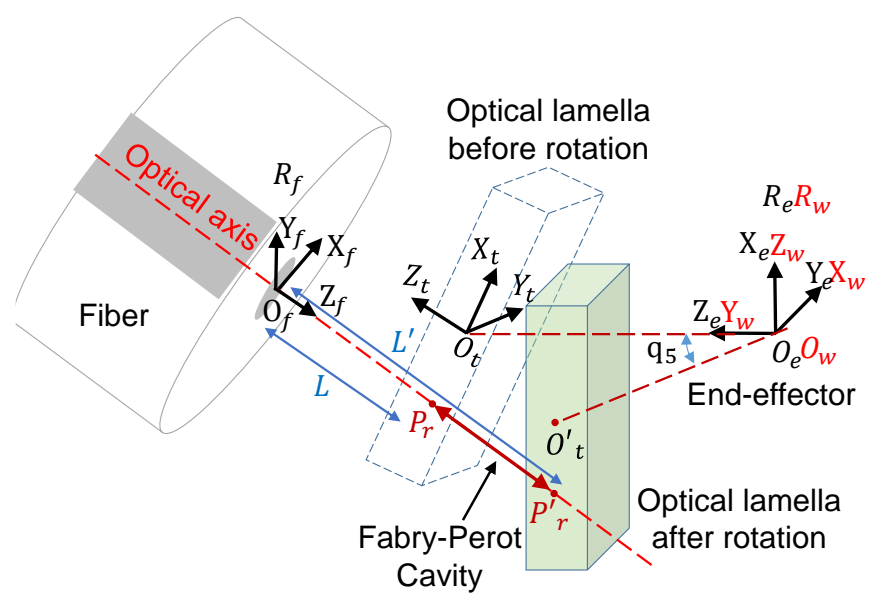

Figure 6. Optical lamella before the new rotation and after the new rotation (around $q_{5}$ ).

When $q_{1}=q_{2}=q_{3}=q_{3}=q_{6}=0$, and by applying two axis-by-axis rotations $q_{4}$ and $q_{5}$, equation 15 can be written as follows:

$$
\begin{gathered}
{ }^{w} T_{t}=\left[\begin{array}{cccc}
\cos \left(q_{5}\right) & 0 & -\sin \left(q_{5}\right) & 0 \\
\sin \left(q_{5}\right) \cos \left(q_{4}\right) & \sin \left(q_{4}\right) & \cos \left(q_{5}\right) \cos \left(q_{4}\right) & 0 \\
\sin \left(q_{5}\right) \sin \left(q_{4}\right) & -\cos \left(q_{4}\right) & \cos \left(q_{5}\right) \sin \left(q_{4}\right) & 0 \\
0 & 0 & 0 & 1
\end{array}\right] . \\
{\left[\begin{array}{cccc}
r & r & a_{e t} & 0 \\
r & r & b_{e t} & 0 \\
r & r & c_{e t} & Z_{e t} \\
0 & 0 & 0 & 1
\end{array}\right]=\left[\begin{array}{cccc}
r & r & a_{w t}^{\prime} & -Z_{e t} \sin \left(q_{5}\right) \\
r & r & b_{w t}^{\prime} & Z_{e t} \cos \left(q_{4}\right) \cos \left(q_{5}\right) \\
r & r & c_{w t}^{\prime} & Z_{e t} \cos \left(q_{5}\right) \sin \left(q_{4}\right) \\
0 & 0 & 0 & 1
\end{array}\right]}
\end{gathered}
$$

The new optical lamella plane equation after rotation with respect to the world frame and as shown in Figure 6 is given:

$$
\begin{gathered}
a_{w t}^{\prime} x+b_{w t}^{\prime} y+c_{w t}^{\prime} z+d_{w t}^{\prime}=0 \\
\left\{\begin{array}{l}
a_{w t}^{\prime}=\cos \left(q_{5}\right) a_{e t}-\sin \left(q_{5}\right) c_{e t} \\
b_{w t}^{\prime}=\cos \left(q_{4}\right) \sin \left(q_{5}\right) a_{e t}+\sin \left(q_{4}\right) b_{e t}-\cos \left(q_{4}\right) \cos \left(q_{5}\right) c_{e t} \\
c_{w t}^{\prime}=\sin \left(q_{4}\right) \sin \left(q_{5}\right) a_{e t}-\cos \left(q_{4}\right) b_{e t}+\cos \left(q_{5}\right) \sin \left(q_{4}\right) c_{e t} \\
d_{w t}^{\prime}=a_{w t}^{\prime} Z_{e t} \sin \left(q_{5}\right)-b_{w t}^{\prime} Z_{e t} \cos \left(q_{4}\right) \cos \left(q_{5}\right) \\
-c_{w t} Z_{e t} \cos \left(q_{5}\right) \sin \left(q_{4}\right)
\end{array}\right.
\end{gathered}
$$

By taking the two optical lamella plane equations in the initial pose and after rotation with respect to the world frame as shown in Figure 6 and the optical axis line equation in the world frame as given in equation (8), the following equation can be obtained.

$$
\left\{\begin{array}{l}
a_{w t} x+b_{w t} y+c_{w t} z+d_{w t}=0 \\
a_{w t}^{\prime} x+b_{w t}^{\prime} y+c_{w t}^{\prime} z+d_{w t}^{\prime}=0
\end{array}\right.
$$

The intersection of the optical axis line with the first optical lamella plane (before rotation) gives:

$a_{w t}\left(a_{w f} L+x_{w f}\right)+b_{w t}\left(b_{w f} L+y_{w f}\right)+c_{w t}\left(c_{w f} L+z_{w f}\right)+d_{w t}=0$

The intersection of the optical axis line with the first optical lamella plane (after rotation) gives:

$a_{w t}^{\prime}\left(a_{w f} L^{\prime}+x_{w f}\right)+b_{w t}^{\prime}\left(b_{w f} L^{\prime}+y_{w f}\right)+c_{w t}^{\prime}\left(c_{w f} L^{\prime}+z_{w f}\right)+d_{w t}^{\prime}=0$

The optical lamella distance change $d L$ along the optical axis after rotation can be deduced as shown follows:

$$
\begin{aligned}
& d L=L^{\prime}-L=\frac{\left(a_{w t} x_{w f}+b_{w t} y_{w f}+c_{w t} z_{w f}+d_{w t}\right)}{a_{w t} a_{w f}+b_{w t} b_{w f}+c_{w t} c_{w f}}- \\
& \frac{\left(a_{w t}^{\prime} x_{w f}+b_{w t}^{\prime} y_{w f}+c_{w t}^{\prime} z_{w f}+d_{w t}^{\prime}\right)}{a_{w t}^{\prime} a_{w f}+b_{w t}^{\prime} b_{w f}+c_{w t}^{\prime} c_{w f}}
\end{aligned}
$$

At the same time this optical lamella distance change on the optical axis after rotation can be measured from the FabryPerot interferometry measure.

The parameters $\left(a_{w t}, b_{w t}, c_{w t}, a_{w f}, b_{w f}, c_{w f}\right)$ have already been identified. By applying different rotations $q_{4}$ and $q_{5}$, we build a system of linear equations, which will permit to calculate the four parameters $\left(Z_{e t}, x_{w f}, y_{w f}, z_{w f}\right)$, the dedicated measurement strategy for identification of $O_{t}\left(0,0, Z_{e t}\right)$ and $O_{f}\left(x_{w f}, y_{w f}, z_{w f}\right)$ is discussed in the next section. 
C. Calculation of the reflection point projection $P_{r}$ on the plane $\left(Y_{t} O_{t} X_{t}\right)$ with respect to the tool frame and the world frame $\left({ }^{t} P_{r x},{ }^{t} P_{r y},{ }^{w} P_{r x},{ }^{w} P_{r y},{ }^{w} P_{r z}\right)$

The Cartesian coordinates of $O_{t}$ and $O_{f}$ in the world frame have been identified, the orientations of the optical lamella plane and the optical axis line with respect to the world frame have also been identified. Hence, the point of intersection between the optical axis and the plane of the optical lamella in the world frame can be calculated by calculating the distance $l$ between the optical lamella and the fiber using equation (29, and then calculating the Cartesian coordinates of the reflection point ${ }^{w} P_{r}\left({ }^{w} P_{r x},{ }^{w} P_{r y},{ }^{w} P_{r z}\right)$ in the world frame using:

$$
\left\{\begin{array}{l}
{ }^{w} P_{r x}=a_{w f} L+x_{w f} \\
{ }^{w} P_{r y}=b_{w f} L+y_{w f} \\
{ }^{w} P_{r z}=c_{w f} L+z_{w f}
\end{array}\right.
$$

The Direct Geometric Model (DGM) of the robot ${ }^{w} T_{e}$ is supposed nominal, therefore when $q_{1}=q_{2}=q_{3}=$ $q_{3}=q_{4}=q_{5}=q_{6}=0$, the Direct Geometric Model (DGM) transformation matrix can be deduced as shown in the following equation:

$$
{ }^{w} T_{e}=\left[\begin{array}{cccc}
1 & 0 & 0 & 0 \\
0 & 0 & 1 & 0 \\
0 & -1 & 0 & 0 \\
0 & 0 & 0 & 1
\end{array}\right]
$$

By replacing the already deduced vector ${ }^{w} P_{r}$ and the transformation matrices ${ }^{e} T_{t}$ and ${ }^{w} T_{e}$, the vector ${ }^{t} P_{r}$ can be deduced as follows:

$$
{ }^{t} P_{r}={ }^{e} T_{t}^{-1} *{ }^{w} T_{e}^{-1} *{ }^{w} P_{r}
$$

In this section, the proposed methodology for extrinsic parameters identification is presented. The next section presents the experimental procedure for robot extrinsic parameters calibration.

\section{EXPERIMENTAL INVESTIGATIONS AND BEHAVIOR OF THE CALIBRATED ROBOT}

The whole experimental procedure of extrinsic parameters calibration includes measurement and identification, parameters integration and validation steps. Each step is presented in detail.

\section{A. Measurement strategy and identification}

There are two adopted measurement strategies that enables to identify two set of different complementary parameters:

1) Measurement strategy for the identification of the coordinates of the unit vector $\vec{Z}_{f}\left(a_{w f}, b_{w f}, c_{w f}\right)$ and the coordinates of the unit vector $\vec{Z}_{t}\left(a_{w t}, b_{w t}, c_{w t}\right)$ :

Based on Fabry-Perot interferometry principle, when the lamella moves along the optical axis, it produces systematically Fabry-Perot interferometry phenomenon and a change of the Fabry-Perot cavity length $(L)$ happens. Therefore, there is a correlation between the FP cavity length $L$ and the interference figure. A slight variation of the FP cavity length can be identified on the interference figure.

Notice: in this case, the orientation of the optical lamella and the fiber has to stay constant, which means only translations are allowed.
The adopted measurement strategy uses different lamella position combinations and translations. For each lamella position along the axis $Z_{1}$ of $q_{1}=(-150,0,150) \mu \mathrm{m}$, a continuous translation of $300 \mu \mathrm{m}$ is applied along $X_{w}$ and a translation of $20 \mu \mathrm{m}$ along $Y_{w}$ axis, this gives 6 equations. For each lamella position along the axis $Z_{2}$ of $q_{2}=(-150,0,150) \mu \mathrm{m}$, a continuous translation of $300 \mu \mathrm{m}$ is applied along $X_{w}$ and $Z_{w}$, this gives other 6 equations. For each lamella position along the axis $Z_{3}$ of $q_{3}=(-150,0,150) \mu \mathrm{m}$, a continuous translation of $300 \mu \mathrm{m}$ is applied along $Z_{w}$ and a translation of $20 \mu \mathrm{m}$ along $Y_{w}$ axis, this gives again other 6 equations. The overall number of non-linear equations is 18 by applying the 3 translations.

For the translation along $X_{w}$ and $Z_{w}$ axes, the sign of $d L$ is required. For this sake, a wavelength scan is used to calculate the distance $L$ between the fiber and the optical lamella before and after each translation along $X_{w}$ and $Z_{w}$ axes. The difference between the measured distance before and after each translation gives the sign of Fabry-Perot measure of the applied translation.

The free spectral range in wavelength $\left(\delta \lambda_{F S R}\right)$ of the FabryPerot cavity in general is given by:

$$
\delta \lambda_{F S R}=\frac{\lambda^{2}}{2 n L}
$$

where $\lambda$ is the vacuum wavelength of light. For a linear cavity, such as the Fabry-Perot, $L$ is the length of the cavity, $n$ is the medium index within the cavity.

And at the same time, the free spectral range in wavelength $\left(\delta \lambda_{F S R}\right)$ can be calculated as follows:

$$
\delta \lambda_{F S R}=\delta T \frac{\lambda_{\text {step }}}{t_{\text {step }}}
$$

where $\lambda_{\text {step }}$ and $t_{\text {step }}$ are the wavelength and time steps respectively. $\delta T$ can be identified from the reflected irradiance as shown in Figure 7 After calculating the free spectral range in wavelength from equation (36, the cavity distance $L$ can be deduced from equation 35 .

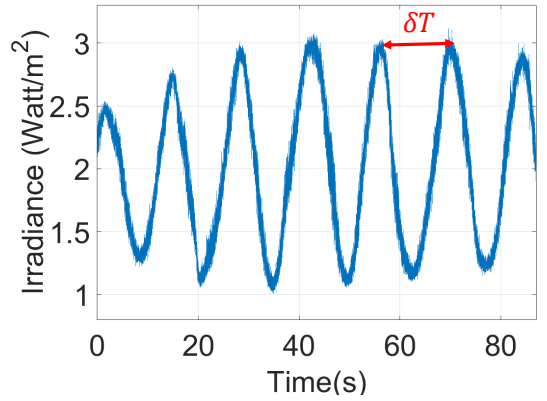

Figure 7. Wavelength scan where $\lambda_{\text {step }}=0.01 \mu \mathrm{m}$ and $t_{\text {step }}=0.01 \mathrm{~s}$.

The resolution of these non-linear equations using nonlinear least-squares solver permits to identify the parameters $\left(a_{w t}, b_{w t}, c_{w t}, a_{w f}, b_{w f}, c_{w f}\right)$, which are the orientations of the optical axis line and the optical lamella plane.

2) Measurement strategy for identification of the Cartesian coordinates of the two origins $O_{t}\left(0,0, Z_{\text {et }}\right)$ and $O_{f}\left(x_{w f}, y_{w f}, z_{w f}\right)$ of the optical lamella and the fiber respectively with respect to the world frame : 


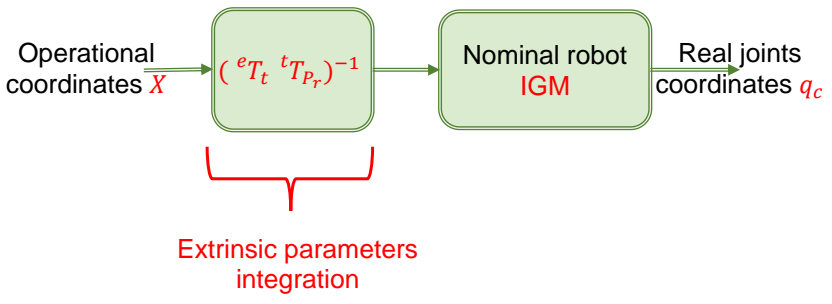

Figure 8. Extrinsic parameters integration.

The adopted measurement strategy uses different combinations of poses. A continuous axis-by-axis rotation is applied around the axis $Z_{4}$ of $q_{4}=-4^{\circ}$ to $4^{\circ}$ for different angles of $q_{5}=-4^{\circ},-2^{\circ}, 0^{\circ}, 2^{\circ}, 4^{\circ}$. Then, a continuous axis-by-axis rotation is applied around the axis $Z_{5}$ of $q_{5}=-4^{\circ}$ to $4^{\circ}$ for different angles of $q_{4}=-4^{\circ},-2^{\circ}, 0^{\circ}, 2^{\circ}, 4^{\circ}$. The rotations are applied based on velocity control. For each continuous rotation, the lamella distance change along the optical axis is measured, each rotation gives 1 equation. Therefore, the final number of equations is 10 .

A system of 10 equations with 4 unknowns is built. The system is overdetermined, since there are more equations than unknowns. The method of ordinary least squares can be used to find an approximate solution to overdetermined systems. For the system $A X=b$, the least squares formula is obtained from the problem $\min \|A X-b\|$, the solution of which can be written as follows: $X=\left(A^{T} A\right)^{-1} A^{T} b$ [25], where $T$ indicates a matrix transpose.

3) Extrinsic parameters integration: The identification of the previously presented parameters leads to build the transformations ${ }^{t} T_{P_{r}},{ }^{w} T_{P_{r}}$ and ${ }^{e} T_{t}$. The integration scheme of the identified extrinsic parameters is shown in Figure 8. The inverse kinematic model of the robot is supposed nominal.

Our objective is to rotate the optical lamella around $X_{f}, Y_{f}$ and $Z_{f}$ axes with a fixed reflection point $P_{r}$, and to translate the optical lamella perpendicular to the optical axis $Z_{f}$. For this sake, in the validation step, a $100 \mu \mathrm{m}$ radius circular translation of the lamella along $X_{f}$ and $Y_{f}$ is applied and a 3 rotations are applied around $X_{f}, Y_{f}$ and $Z_{f}$ axes (see Figure 5 for frames assignment).

\section{B. Experimental setup}

The experimental set up proposed for 6-DOF automated positioning of optical components is schematically depicted in Figure 9 A 6 DOF serial micro-positioning robot, is proposed to position the optical lamella and control its poses. Further is referred as a robot. An other $\mathrm{XYZ}$ manual positioner is fixed in front of the 6 DOF robot, it is used to hold the fiber ferrule and pre-position it. The whole system is referred as photo-robotic system.

The photo-robotic system is mounted on an anti-vibration table. The 6-DOF robot is assembled with minimum geometric errors based on one (XYZ) translation stage followed by a bracket, which is made specifically to join the (XYZ) translation stage $\left(q_{1}, q_{2}, q_{3}\right)$ to a set of two gomiometers $\left(q_{4}, q_{5}\right)$ and

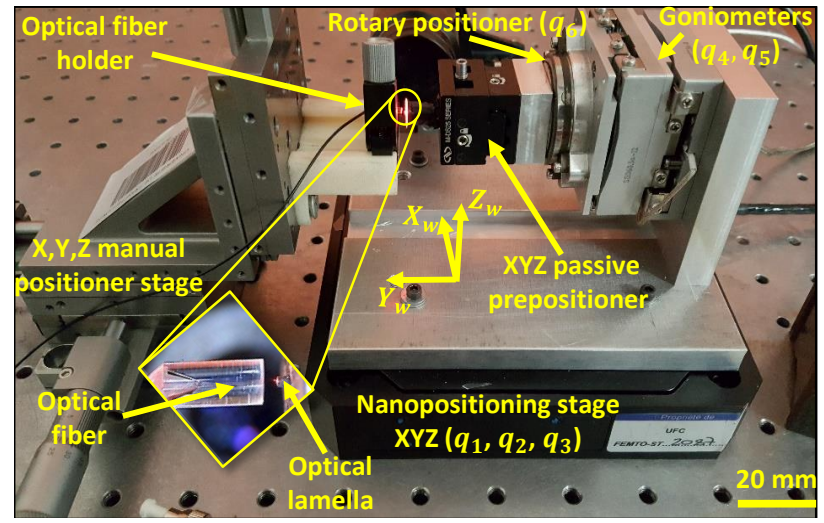

Figure 9. 6-DOF Photo-robotic experimental platform.

a rotary positioner $\left(q_{6}\right)$ (see Figure 9). The two gomiometers are used for rotation about $\mathrm{X}$-axis and $\mathrm{Z}$-axis and the rotary positioner for rotation around Y-axis. A passive XYZ prepositioner is mounted after the rotary positioner for coarse adjustment of the rotation center of the whole assembled robot.

The (XYZ) translation stage is a PIMars P-563.3CD equipped with a E-712 controller. The two goniometers are SGO-60.5 and SGO-77.5, which use the stick-slip principle and is controlled by MCS-3D unit. The SGO-60.5 goniometer's center of rotation is $60.5 \mathrm{~mm}$. The SGO-77.5 goniometer's center of rotation is extended to $77.5 \mathrm{~mm}$. Rotary positioner is SmarAct SR-3610-S which uses the piezo principle and is controlled by MCS-3D unit. All the micro-positioning stages are equipped with internal sensors and are closed-loop controlled in joint layers already.

The optical set up consists in a laser light source (wavelength $\lambda=1560 \mathrm{~nm}$ ) connected to a circulator and then to the fiber ferrule. This configuration permits to align and assemble the optical lamella to the fiber. The reflected irradiance from optical lamella through the fiber ferrule is the closed loop information for the robotic micro-manipulator poses control. This set up permits the acquisition of irradiance signal from the reflected light from the optical lamella surface, returning back through the fiber ferrule and then through the beam circulator. Fabry-Perot measurement is used to control the relative position between fiber ferrule/optical lamella actively.

The next section studies and investigates the optical performances after having applied robot extrinsic parameters calibration approach.

\section{Behavior study of the calibrated robot extrinsic parameters}

Figure 10 represents axis-by-axis rotations $q_{4}, q_{5}$ and $q_{6}$ around $Z_{4}, Z_{5}$ and $Z_{6}$ axes respectively before calibration, and square translation on the optical lamella plane before calibration. The fringes (peaks) on the optical irradiance are due to Fabry-Perot cavity variation along the optical axis. This proves the existence of some geometric errors, that can be extrinsic and/or intrinsic. The type of optical irradiance in Figure 10 appears in active positioning of optical components, which leads to local maximum problem. As a result, it is a time consuming especially for multi-DOF alignment process to reach maximum optical power. 

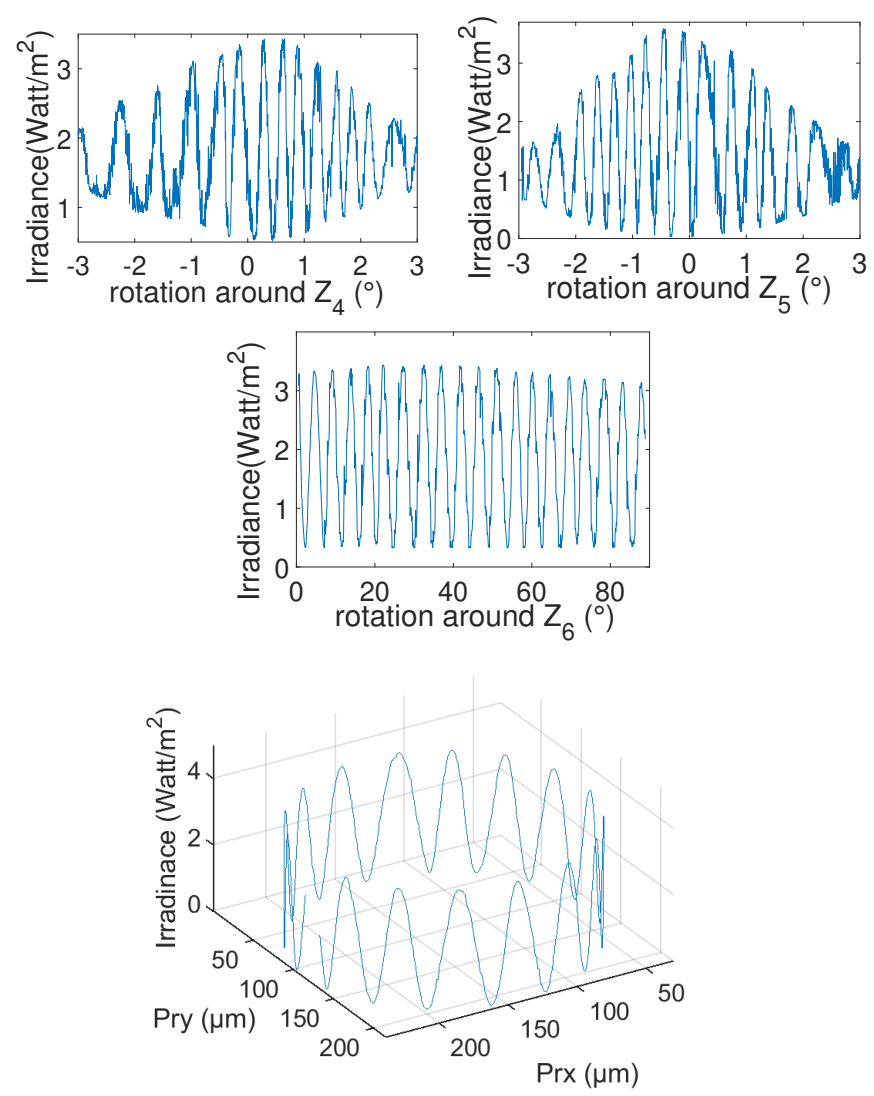

Figure 10. Rotations around $Z_{4}, Z_{5}$ and $Z_{6}$ axes and square translation on the optical lamella plane before calibration.

The objective of robot geometric parameters calibration is to realize 6-DOF positioning without Fabry-Perot cavity variation on the optical axis i.e, realizing rotations around $X_{f}, Y_{f}$ and $Z_{f}$ axes and translation along the optical lamella plane without translating along the optical axis (The reflection point on the lamella plane is kept fixed). For this sake, first extrinsic parameters calibration of the 6-DOF robot is realized. The duration of the measurement and identification phase is about 30 minutes. After achieving measurement and identification phases, the identified extrinsic parameters are obtained and shown in Table II Then, integration of the obtained extrinsic parameters is performed as shown in Figure 8, where the inverse kinematic model of the robot is supposed nominal. A validation test is then realized which aims at quantifying the performances of the robot after calibration of the extrinsic parameters.

Figure 11 represents the obtained results after having integrated extrinsic parameters for, 3 rotations around $X_{f}, Y_{f}$ and $Z_{f}$, after identified the reflection point projection on the lamella $P_{r}$. For rotations around $X_{f}, Y_{f}$, the corresponding optical irradiance and the 3 translational joints are plotted (since they are the only joints which intervene for these two rotations). For rotations around $Z_{f}$, the corresponding optical irradiance and the 3 translational joints and the 2 rotational joints (joint 4 and 5) are plotted.

We can notice that after having calibrated robot extrinsic parameters, the joints intervene in order to move the rotation center of robot to the reflection point of the laser spot $P_{r}$ and
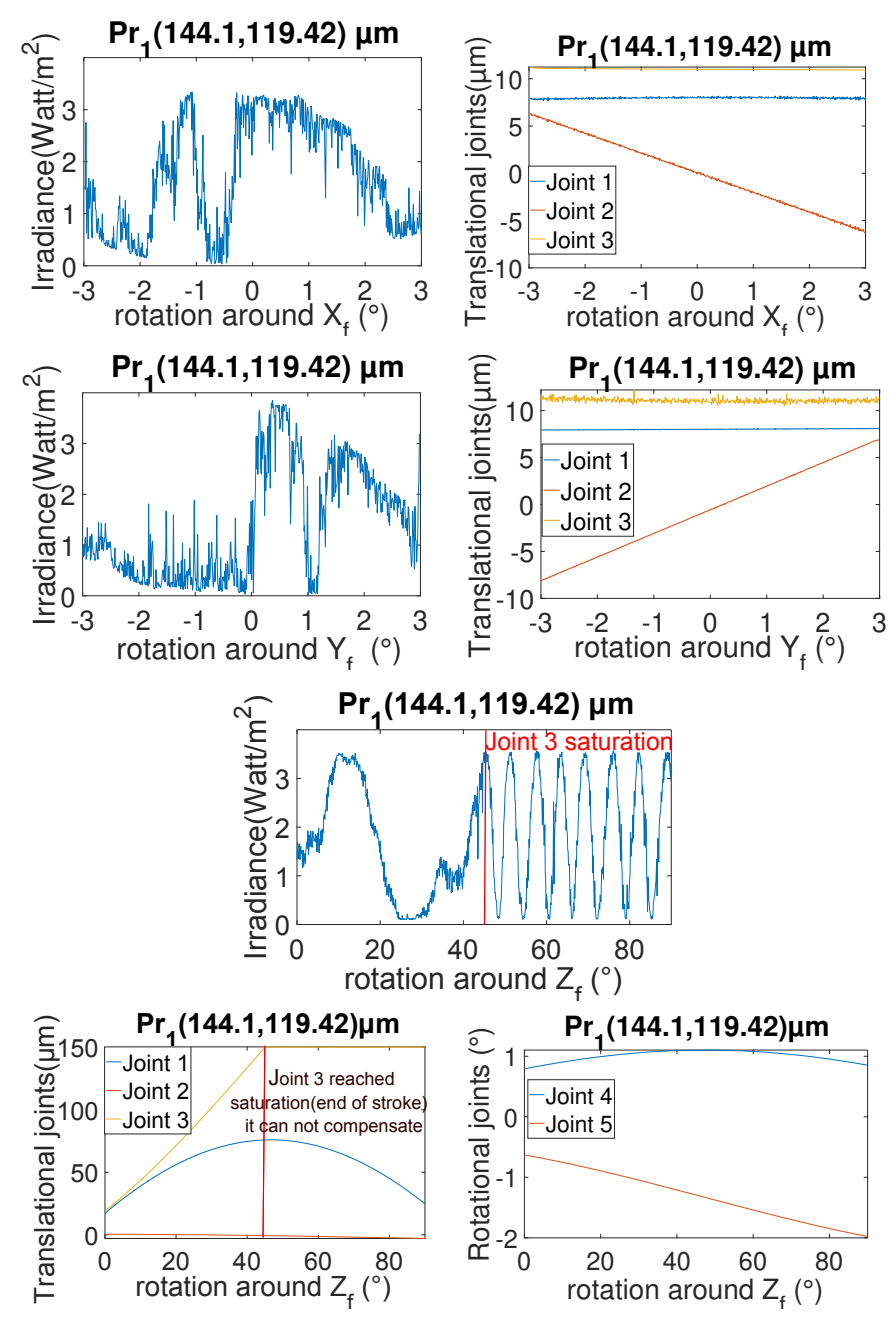

Figure 11. 3 rotations around $X_{f}, Y_{f}$ and $Z_{f}$ axes when the reflection point projection is the reflection point $P_{r}$, after robot extrinsic parameters calibration.

maintain rotation at that point (reflection point $P_{r}$ ).

For rotations around $X_{f}$ and $Y_{f}$, there exist some fringes (oscillations) on the optical irradiance, which are interpreted by the existence of small Fabry-Perot cavity variation during the two rotations. The estimated Fabry-Perot cavity variation for the two rotations around $X_{f}$ and $Y_{f}$ of rotation range of $6^{\circ}$ is $1.56 \mu \mathrm{m}$.

For a rotation around $Z_{f}$, the translational and rotational joints intervene in order to maintain the rotation at the reflection point $P_{r}$. There exist some fringes on the optical irradiance, which are interpreted by the existence of small Fabry-Perot cavity variation during the rotation. When at least one translational joint is saturated, the frequency of the fringes increase, which represent an increase in Fabry-Perot cavity variation during the rotation. The estimated Fabry-Perot cavity variation for the rotation around $Z_{f}$ is $0.858 \mu \mathrm{m}$ for a rotation range of $45.5^{\circ}$.

Figure 12 shows the optical irradiance corresponding to circular translations respectively along the optical lamella plane before and after extrinsic parameters calibration. We can notice that the number of fringes decrease considerably after robot extrinsic parameters calibration. Before calibration, a 
Table II

IDENTIFIED EXTRINSIC PARAMETERS.

\begin{tabular}{cccccccccccc}
\hline Parameters & $\beta_{\text {et }}$ & $\theta_{\text {et }}$ & $\beta_{w f}$ & $\theta_{w f}$ & $Z_{\text {et }}$ & $x_{w f}$ & $y_{w f}$ & $z_{w f}$ & ${ }^{w} P_{r x}$ & ${ }^{w} P_{r y}$ & ${ }^{w} P_{r z}$ \\
Values & $0.744^{\circ}$ & $-0.595^{\circ}$ & $0.732^{\circ}$ & $-0.520^{\circ}$ & $1.52 \mu \mathrm{m}$ & $112.40 \mu \mathrm{m}$ & $900.0 \mu \mathrm{m}$ & $113.3 \mu \mathrm{m}$ & $155.2 \mu \mathrm{m}$ & $-2.7 \mu \mathrm{m}$ & $-111.34 \mu \mathrm{m}$ \\
\hline
\end{tabular}

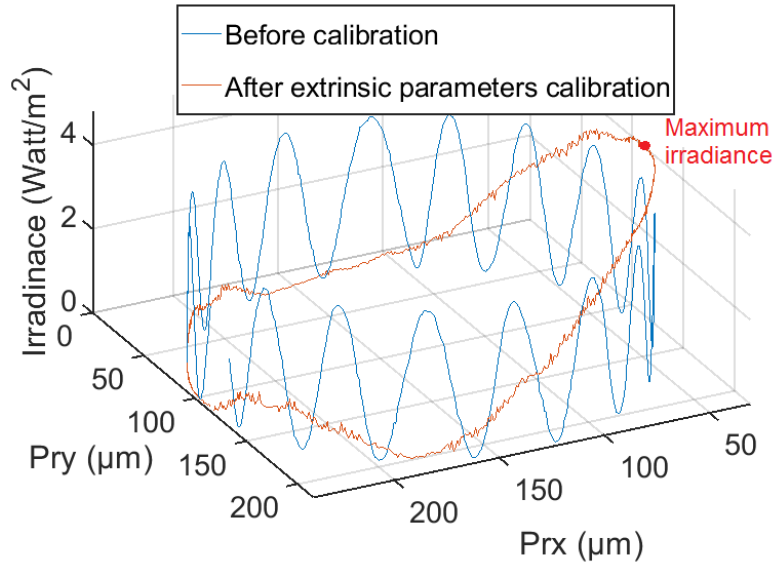

Figure 12. The optical irradiance corresponding to circular translation on the optical lamella plane before and after extrinsic parameters calibration.

circular translation of a circumference of $628.32 \mu \mathrm{m}$ produced $12.87 \mu \mathrm{m}$ Fabry-Perot cavity variation along the optical axis. After calibration, the Fabry-Perot cavity variation along the optical axis is about $390 \mathrm{~nm}$. Hence, From the yielded optical irradiance from the circular translation, the optimal position for the optical lamella can be identified to achieve the maximum optical irradiance as highlighted in red color.

\section{CONCLUSION}

The paper deals with an integrated optics system achieved through highly accurate multi-DOF robotic active positioning. For this sake, two optical components were chosen, a fiber ferrule and an optical lamella. Fabry-Perot interferometry principle was used to provide a rapid and high accurate measurement.

A photo-robotic approach was proposed for robot extrinsic parameters calibration. This approach relates a 1-D relative distance with a multi-DOF robot motion in order to identify extrinsic geometric parameters based on a photo-robotic calibration model. A dedicated measurement strategy was proposed for high measurement accuracy, where the 1-D relative distance measure is achieved based on the highly accurate 1-D Fabry-Perot interferometry measurement.

A photo-robotic platform dedicated for optical components 6-DOF nano-positioning and assembly was proposed. A 6DOF serial robot is proposed, which is built based on highly accurate nano-positioning stages commonly used for micromanipulation.

Applying the proposed photo-robotic extrinsic parameters calibration reduced considerably Fabry-Perot cavity variation during rotations around $X_{f}, Y_{f}$ and $Z_{f}$, after identified the reflection point of the laser spot. We have achieved a FabryPerot cavity variation of about $1.56 \mu \mathrm{m}$ for a rotation range of $6^{\circ}$ around $X_{f}$ and $Y_{f}$ and a Fabry-Perot cavity variation of about $0.858 \mu \mathrm{m}$ for a rotation range of $45.5^{\circ}$ around $Z_{f}$. Applying circular translation of $100 \mu \mathrm{m}$ radius along the optical lamella plane after robot extrinsic parameters calibration reduced also considerably Fabry-Perot cavity variation. We have achieved a Fabry-Perot cavity variation of about 390 nm during a circular translations. From the yielded optical irradiance from the circular translation, the optimal position for the optical lamella can be identified to achieve the maximum optical irradiance.

\section{ACKNOWLEDGMENT}

These works have been funded by the Franche-Comté region, nano-roboptic project supported by the EUR EIPHI Graduate School (ANR-17-EURE-0002), ANR CEPAGE ANR-16-CE24-0024 and by the French RENATECH network through its FEMTO-ST technological facility.

\section{REFERENCES}

[1] J. Agnus, N. Chaillet, C. Clévy, S. Dembélé, M. Gauthier, Y. Haddab, G. Laurent, P. Lutz, N. Piat, K. Rabenorosoa et al., "Robotic microassembly and micromanipulation at femto-st," Journal of Micro-Bio Robotics, vol. 8, no. 2, pp. 91-106, 2013.

[2] L. Zimmermann, G. B. Preve, T. Tekin, T. Rosin, and K. Landles, "Packaging and assembly for integrated photonics-a review of the epixpack photonics packaging platform," IEEE Journal of Selected Topics in Quantum Electronics, vol. 17, no. 3, pp. 645-651, 2011.

[3] O. Benson, "Assembly of hybrid photonic architectures from nanophotonic constituents," Nature, vol. 480, no. 7376, p. 193, 2011.

[4] R. Takigawa, E. Higurashi, T. Suga, and T. Kawanishi, "Passive alignment and mounting of linbo _3 waveguide chips on si substrates by low-temperature solid-state bonding of au," IEEE Journal of Selected Topics in Quantum Electronics, vol. 17, no. 3, pp. 652-658, 2011.

[5] Y. Zheng and J.-a. Duan, "Alignment algorithms for planar optical waveguides," Optical Engineering, vol. 51, no. 10, pp. 103 401-1, 2012.

[6] W. Sassen, V. Henneken, M. Tichem, and P. Sarro, "An improved in-plane thermal folded v-beam actuator for optical fibre alignment," Journal of Micromechanics and Microengineering, vol. 18, no. 7, p. 075033, 2008.

[7] B. Li, H. Wirz, and A. Sharon, "Optimizing fiber coupling with a quasipassive microoptical bench," Journal of microelectromechanical systems, vol. 14, no. 6, pp. 1339-1346, 2005.

[8] J. F. C. van Gurp, M. Tichem, U. Staufer, and J. Zhao, "Passive photonic alignment with submicrometer repeatability and accuracy," Components, Packaging and Manufacturing Technology, IEEE Transactions on, vol. 3, no. 11, pp. 1971-1979, 2013.

[9] H. S. Yang, C. Zhang, and M. S. Bakir, "A self-aligning flip-chip assembly method using sacrificial positive self-alignment structures," IEEE Transactions on Components, Packaging and Manufacturing Technology, vol. 6, no. 3, pp. 471-477, 2016.

[10] T. Barwicz, Y. C. Martin, and J.-W. Nah, "Three dimensional selfalignment of flip chip assembly using solder surface tension during solder reflow," Mar. 28 2017, uS Patent 9,606,308.

[11] A. V. Kudryavtsev, G. J. Laurent, C. Clévy, B. Tamadazte, and P. Lutz, "Stereovision-based control for automated moems assembly," in 2015 IEEE/RSJ International Conference on Intelligent Robots and Systems (IROS). IEEE, 2015, pp. 1391-1396. 
[12] C. Clévy, I. Lungu, K. Rabenorosoa, and P. Lutz, "Positioning accuracy characterization of assembled microscale components for micro-optical benches," Assembly Automation, vol. 34, no. 1, pp. 69-77, 2014.

[13] M. Carminati, S. Grillanda, P. Ciccarella, G. Ferrari, M. J. Strain, M. Sampietro, A. Melloni, and F. Morichetti, "Fiber-to-waveguide alignment assisted by a transparent integrated light monitor," IEEE Photonics Technology Letters, vol. 27, no. 5, pp. 510-513, 2015.

[14] P. Shiakolas, K. Conrad, and T. Yih, "On the accuracy, repeatability, and degree of influence of kinematics parameters for industrial robots," International journal of modelling and simulation, vol. 22, no. 4, pp. 245-254, 2002.

[15] W. Veitschegger and C.-H. Wu, "Robot accuracy analysis based on kinematics," IEEE Journal on Robotics and Automation, vol. 2, no. 3, pp. 171-179, 1986.

[16] L. Clark, B. Shirinzadeh, Y. Tian, and D. Oetomo, "Laser-based sensing, measurement, and misalignment control of coupled linear and angular motion for ultrahigh precision movement," IEEE/ASME Transactions on Mechatronics, vol. 20, no. 1, pp. 84-92, 2015.

[17] C. Clévy, M. Rakotondrabe, and N. Chaillet, Signal measurement and estimation techniques for micro and nanotechnology. Springer Science \& Business Media, 2011.

[18] H. Bettahar, A. Caspar, C. Clévy, N. Courjal, and P. Lutz, "Photorobotic positioning for integrated optics," IEEE Robotics and Automation Letters, vol. 2, no. 1, pp. 217-222, 2016.

[19] J. Denavit, "A kinematic notation for low pair mechanisms based on matrices," ASME J. Appl. Mech., vol. 22, pp. 215-221, 1955.

[20] R. Ibarra and N. Perreira, "Determination of linkage parameter and pair variable errors in open chain kinematic linkages using a minimal set of pose measurement data," Journal of Mechanisms, Transmissions, and Automation in Design, vol. 108, no. 2, pp. 159-166, 1986.

[21] W. Khalil and J. Kleinfinger, "A new geometric notation for open and closed-loop robots," in Robotics and Automation. Proceedings. 1986 IEEE International Conference on, vol. 3. IEEE, 1986, pp. 1174-1179.

[22] S. Hayati and M. Mirmirani, "Improving the absolute positioning accuracy of robot manipulators," Journal of Robotic Systems, vol. 2 no. 4 , pp. 397-413, 1985

[23] W. Khalil and E. Dombre, Modeling, identification and control of robots. Butterworth-Heinemann, 2004.

[24] G. G. Slabaugh, "Computing euler angles from a rotation matrix," Retrieved on August, vol. 6, no. 2000, pp. 39-63, 1999.

[25] S. Wold, A. Ruhe, H. Wold, and W. Dunn, III, "The collinearity problem in linear regression. the partial least squares (pls) approach to generalized inverses," SIAM Journal on Scientific and Statistical Computing, vol. 5 , no. 3, pp. 735-743, 1984

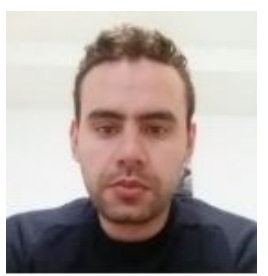

Houari Bettahar graduated from the Institut de Génie Électrique et Électronique (IGEE), Boumerdes, Agérie in 2011 received his Master degree of control system and information technology in 2014 and his Ph. D. degree in Automatic Control and robotics in 2019. Since 2018, he has been an teaching and research Officer at the University of Franche-Comte, Besançon, France working in the AS2M (Automatic Control and MicroMechatronic Systems) department of FEMTO-ST Institute. His research interests are the control of microrobotic and micro-assembly systems oriented to integrated optics.

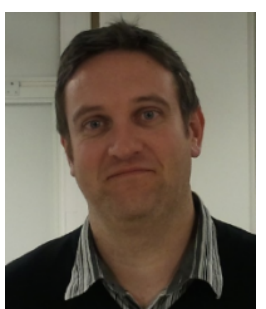

Olivier Lehmann received the Engineer degree (M.S. degree) in automation, mechanics and computer sciences from ENSMM, Besançon, France, in 2000. He was an engineer in charge of technology transfer at the Institut de Productique and then at the Institut Pierre Vernier. In this context, he developed numerous robotics solutions dedicated to SMEs for 13 years and helped SMEs in their development $\mathrm{He}$ is currently research engineer at FEMTO-ST. He is in charge of the development of several micronanorobotic stations in vacuum or non-vacuum environment. His scientific fields are robotics, control, vision and the development of integrated solutions.

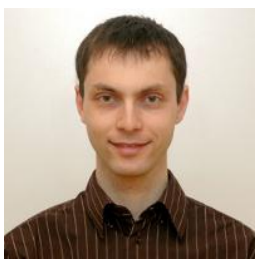

Cédric Clévy received his $\mathrm{Ph}$. D. degree in Automatic Control and computer Sciences in 2005. Since 2006, he has been an Associate Professor at the University of Franche-Comte, Besançon, France working in the AS2M (Automatic Control and MicroMechatronic Systems) department of FEMTOST Institute. His research interests are the design, modeling and control of micro and nano-robotic systems for the characterisation, manipulation and assembly at micro and nanoscales. Cédric Clévy currently leads the "Micro and Nano Robotics" research team of the FEMTO-ST Institute and co-leads the thematic research group "multiscale manipulation" of the Robotics Group of Research from the CNRS - National Research Center. He is also an active member of the IEEE/RAS technical committee for "Micro/Nano Robotics and Automation" and currently serving as Associate Editor for IEEE T-ASE and Technical Editor for IEEE/ASME T-Mech

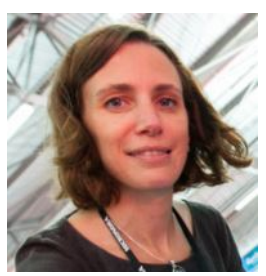

Nadège Courjal received her PhD degrees in "Engineering science" in 2002 and she became Associate Professor at the FEMTO-ST Institute in Besançon in 2004. Her field of expertise relies on integrated optics and photonics, and most particularly electrooptic materials and lithium niobate. She also works on high resolution characterization of photonic components and nanostructures. Amongst her R\&D activities in integrated optics and photonics, she has worked on the production of electro-optic sensors with Kapteos, chipscale spectrometers with Resolution Spectra, and low-chirp Mach-Zehnder Intensity modulators with iXBlue PSD. In the framework of academic research, she has had 3 DGA REI, RAPID $\&$ Astrid projects, and she is or has been the leader of 11 national projects, supported by ANR, BPI, Franche-Comté Region or SATT; she was also the project leader of one ActPhast project in 2016.

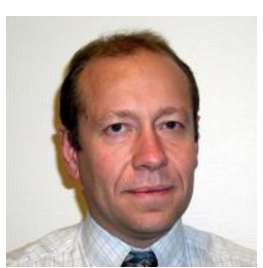

Philippe Lutz joined the University of FrancheComté, Besancon, as Professor in 2002. He was the head of the research group "Automated Systems for Micromanipulation and Micro-assembly" of the AS2M department of FEMTO-ST Institute from 2005 to 2011. He was the Director of the $\mathrm{PhD}$ graduate school of Engineering science and Microsystems with more than $400 \mathrm{PhD}$ students from 2011 to February 2017, and he is currently the head of the Doctoral College. Since January 2017, he is the director of the AS2M Research department of FEMTO-ST. His research activities at FEMTO-ST are focused on the design and control of micro-nano systems, microgrippers, micro-nano robots, feeding systems and micro-nano manipulation, assembly stations, and Optimal design and control of piezoelectrically actuated compliant structures. P. Lutz received several awards of IEEE, authored over 90 refereed publications (50 in high standard journals), serves as associate editor for the IEEE T-ASE and as Technical Editor for the IEEE/ASME T-Mech, is member of several steering committees and is member of the IEEE Robotics and Automation Society (RAS) Committee on Micro-Nano Robotics. 